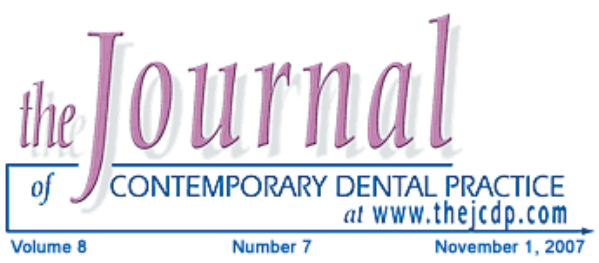

\title{
A Method for Stabilizing a Lingual Fixed Retainer in Place Prior to Bonding
}

\section{Sulaiman AI-Emran, BDS, MSc, PhD; Rakan Barakati, BDS, MSc}

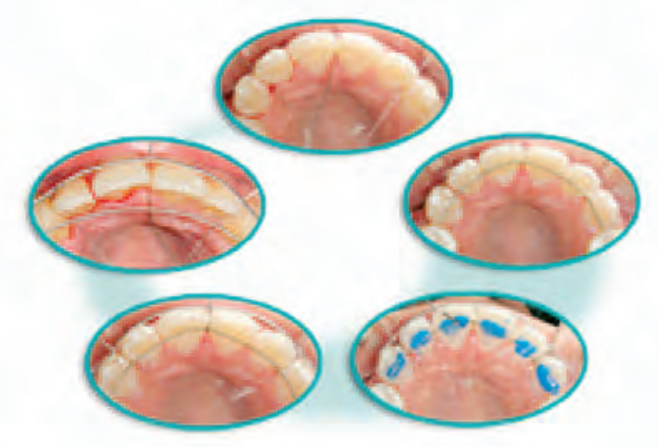

Abstract

Aim: The objective of this article is to present a simple technique for stabilizing a lingual fixed retainer wire in place with good adaptation to the teeth surfaces and checking for occlusal interferences prior to the bonding procedure.

Background: Bonding of an upper or lower fixed lingual retainer using stainless steel wires of different sizes and shapes is a common orthodontic procedure. The retainer can be constructed in a dental laboratory, made at chair side, or it can be purchased in prefabricated form. All three ways of creating a fixed retainer are acceptable. However, the method of holding the retainer wire in place adjacent to the lingual surfaces of the teeth before proceeding with the bonding process remains a problem for some practitioners.

Report: The lingual fixed retainer was fabricated using three pieces of .010" steel ligature wire which were twisted into a single strand wire. Another four to five 0.010 " pieces of steel ligature wires were twisted in the same way to serve as an anchor wire from the labial side of the teeth. The retainer wire was bonded using the foible composite.

Summary: The technique presented here for stabilizing the retainer wire prior to bonding provides good stabilization, adaptation, and proper positioning of the retainer wire while eliminating contamination of etched surfaces which might arise during wire positioning before bonding. This technique also allows the clinician the opportunity to check the occlusion and adjust the retainer wire to avoid occlusal interference prior to bonding maxillary retainers. This same clinical strategy can be used to stabilize wires for splinting periodontally affected teeth and traumatized teeth.

Keywords: Lingual retainer, fixed retainer, bonded retainer

Citation: Al-Emran S, Barakati R. A Method for Stabilizing a Lingual Fixed Retainer in Place Prior to Bonding. J Contemp Dent Pract 2007 November; (8)7:108-113.

(C) Seer Publishing 


\section{Introduction}

Bonding of an upper or lower fixed lingual retainer is a common procedure carried out in orthodontic practices. ${ }^{1-4}$ Stainless steel wires of different sizes and shapes are the most frequently used material for fixed orthodontic retainers. ${ }^{2,5-6}$ The retainer can be constructed in a dental laboratory, made at chair side, or it can be purchased in prefabricated form. All three methods of creating a fixed retainer are acceptable. However, the method of holding the retainer wire in place adjacent to the lingual surfaces of the teeth before proceeding with the bonding process remains a problem for some practitioners.

A simple chair side technique of fabricating and stabilizing a lingual retainer before bonding utilizing the existence of a fixed orthodontic appliance before debonding was presented by the author in $1997 .^{7}$ The technique utilized three strands of 0.010 " stainless steel ligature wire which were twisted into a single strand and adapted to the lingual surface of the anterior teeth. The retainer wire was then stabilized and tied against the lingual surface of the lower anterior teeth using another three pieces of 0.010 " stainless steel ligature wire which were passed below the lingual retainer wire and the contact points of teeth. Here the fixed orthodontic appliance served as a labial anchor to which the retainer wire was tied to hold it against the lingual surfaces of the teeth surfaces before bonding was carried out. However, the need arises for bonding a fixed retainer for some patients who are not wearing fixed orthodontic appliances. In these patients the stabilization of the retainer wire in place is rather difficult and the bonding procedure often becomes challenging.

The aim of this article is to introduce a simple method that enables clinicians to stabilize the retainer wire in place with good adaptation against the lingual surfaces of the anterior teeth and check for any occlusal interference before the bonding procedure is carried out.

\section{Technique}

The steps for the fabrication and stabilization of lingual fixed retainers are as follows:

1. The retainer wire is created by holding three pieces of .010" steel ligature wire at both ends with Mathu forceps and twisting them into a single strand wire (Figure 1).

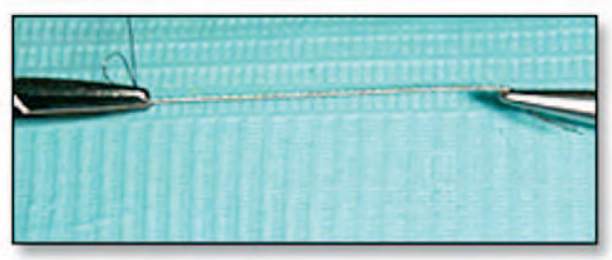

Figure 1. Three 0.010" stainless steel ligature wire twisted into one strand to form the retainer wire.

2. The wire is then curved gently, measured in the patient's mouth, and cut to the desired length (Figure 2).

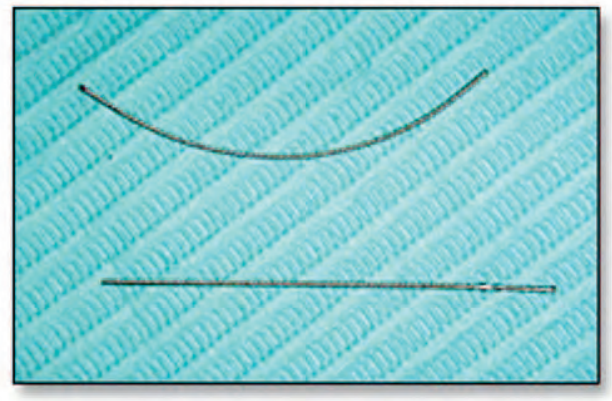

Figure 2. The retainer wire is gently curved before use.

3. Another four to five pieces of 0.010 " steel ligature wire are twisted in the same way to serve as an anchor wire from the labial side of the teeth.

4. The lingual surfaces of the teeth are pumiced, rinsed with water, and dried with compressed air (Figure 3).

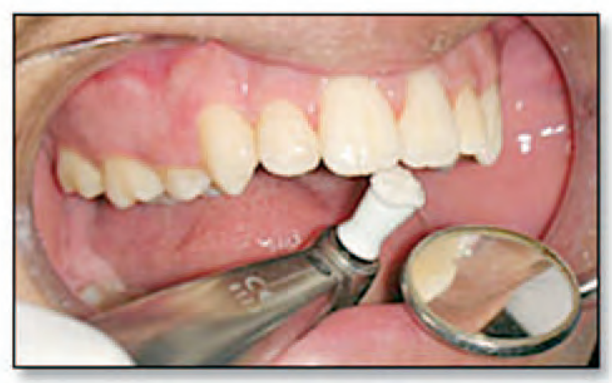

Figure 3. Pumicing of lingual surfaces of teeth to be fixed retained.

5. Three pieces of ligature wire $4-5 \mathrm{~cm}$ long are passed above the contact points of the central incisors, the lateral incisors, and the canines (Figure 4). 


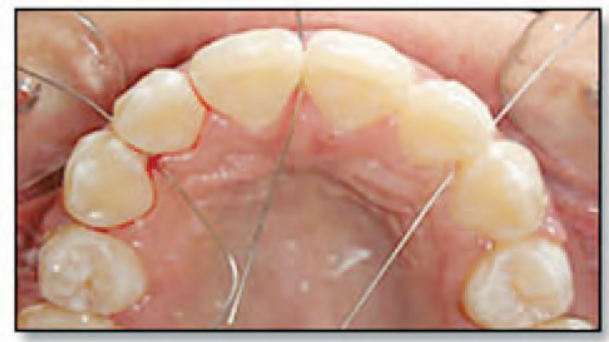

Figure 4. Three pieces of ligature wire 4-5 $\mathrm{cm}$ long are passed above the contact point of the central incisors, lateral incisors, and canines.

6. The retainer wire is placed in the lingual side of the teeth, the anchor wire is placed in the labial side of the teeth, and the two are tied against each other using the three pieces of ligature wire which are inserted gingival to the contact points of teeth (Figures 5 and 6). In cases with open contacts between the teeth, the anchor wire can be bonded to the labial surface of the teeth at both ends to stabilize it during tie up of the retainer wire.

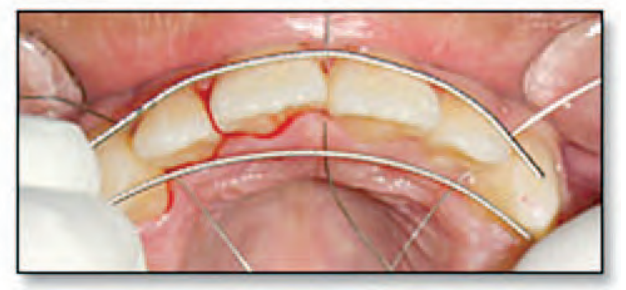

Figure 5. The retainer wire is positioned on the lingual surface of the teeth and the anchor wire is placed on the labial surface of the teeth.

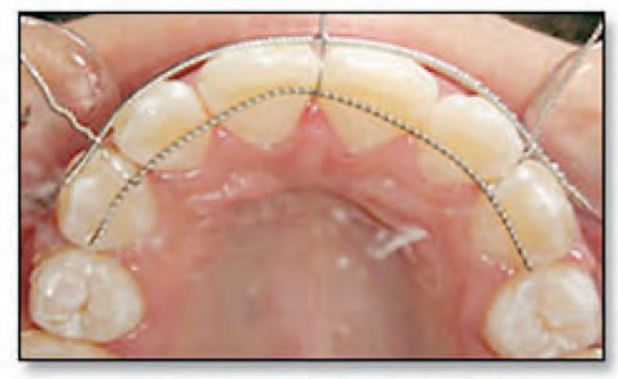

Figure 6. The retainer wire and anchor wire are tied against each other using three pieces of ligature wire inserted gingival to the contact points of teeth.

7. The retainer wire is now adapted to the lingual surfaces of the anterior teeth, if needed, using an amalgam condenser (Figure 7).

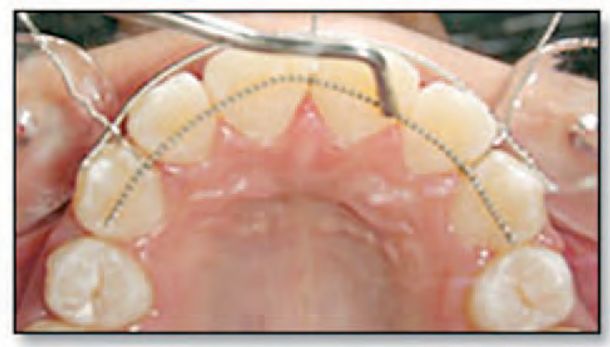

Figure 7. Adaptation and adjustment of the retainer wire in place using an amalgam condenser.

8. If the retainer is done for the upper jaw, the bite should be checked at this stage and the retainer wire adjusted using an amalgam condenser to avoid occlusal interferences (Figure 8).

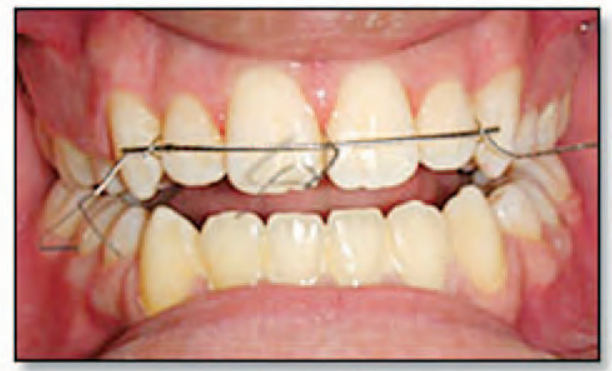

Figure 8. Bite check for occlusal interference.

9. The lingual surfaces of the anterior teeth are etched and the retainer wire is bonded to them using the foible composite (Figures 9 and 10).

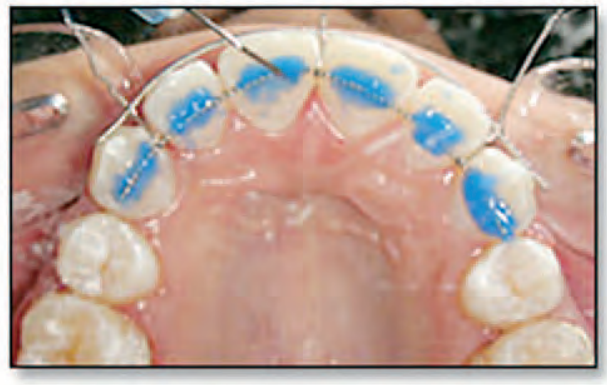

Figure 9. Acid etching of teeth surfaces.

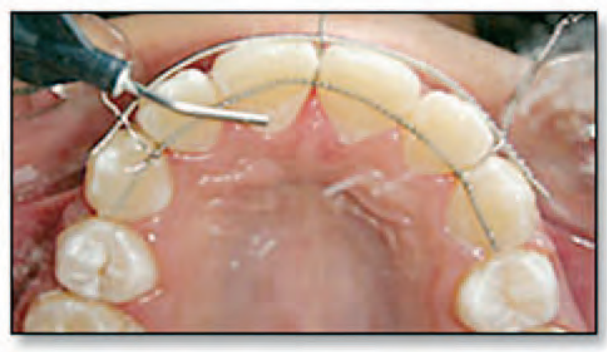

Figure 10. Retainer wire is bonded to the lingual surfaces of the anterior teeth using the foible composite. 
10.Upon completion of bonding the three pieces, ligature wire holding the anchor wire are cut and removed along with the anchor wire and the final evaluation of the occlusion is performed (Figures 11 and 12).

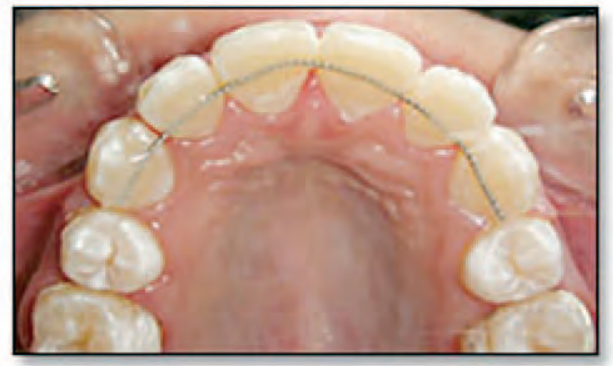

Figure 11. Upper bonded fixed retainer in place.

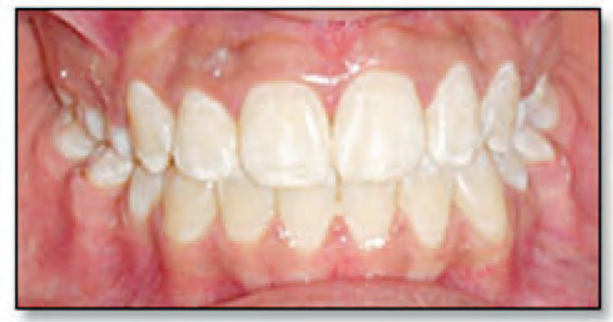

Figure 12. Patient in occlusion with the labial anchor wire removed.

\section{Summary}

The technique presented here for stabilizing the retainer wire prior to bonding provides the following advantages:

1. Favorable stabilization, adaptation, and proper positioning of the retainer wire.

2. Eliminates contamination of etched surfaces which might arise during wire positioning before bonding.

3. Creates the opportunity to check the bite and adjust the retainer wire to avoid occlusal interference before bonding upper retainers.

4. The technique can be used to stabilize wires for splinting teeth compromised periodontally or by trauma. 


\section{References}

1. Zachrisson BU. Clinical experience with direct bonded orthodontic retainers. Am J Orthod. 1977; 71:440-448.

2. Cohen SM, Cassalia B, Hayes RC. Technique Clinic: Direct-bonded lingual retainer with a new twist. J Clin Orthod. 1994; 28:538.

3. Zachrisson BU. Third-generation mandibular bonded lingual 3-3 retainer. J Clin Orthod. 1995; 29:39-48.

4. McNamara TG, McNamara T, Sandy RJ. A new approach to incisor retention- the lingual spur retainer. Br J Orthod. 1996; 23:199-201.

5. Knierim RW. The invisible lower cuspid to cuspid retainer. Angle Orthod. 1973; 43:218-220.

6. Zachrisson BU. The bonded lingual retainer and multiple spacing of anterior teeth. Swed Dent J Suppl. 1982; 15:247-255.

7. Al-Emran S, Hashim H. A chairside bonded lingual retainer. J Clin Orthod. 1997; 31:358-360.

\section{About the Authors}

\section{Sulaiman Al-Emran, BDS, MSc, PhD}
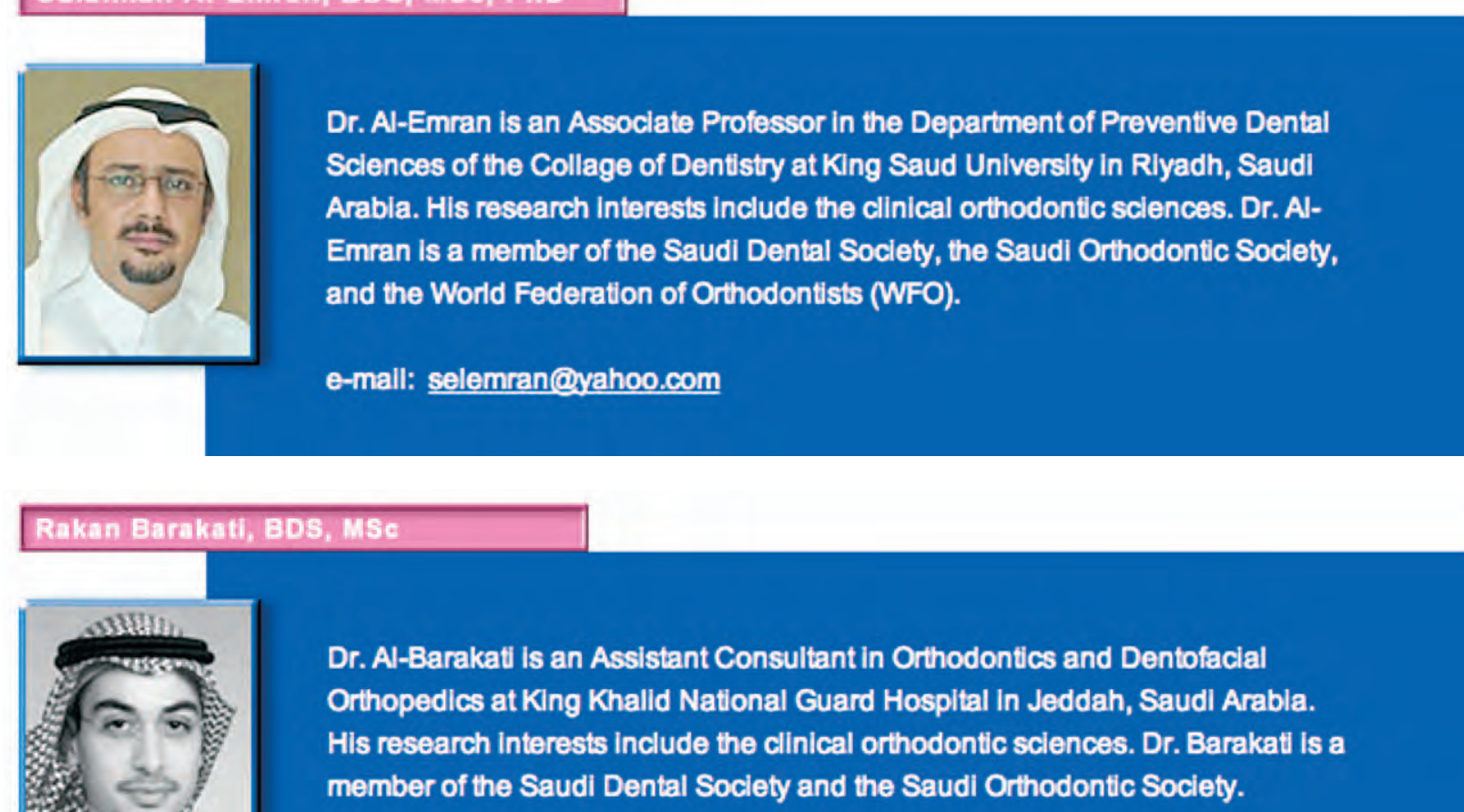

e-mall: dr.rakahn@gmall.com 